\begin{tabular}{|c|l|}
\hline Title & $\begin{array}{l}\text { Formation conditions of coarse columnar austenite grain structure in peritectic carbon steels by the discontinuous grain } \\
\text { growth mechanism }\end{array}$ \\
\hline Author(s) & Ohno, Munekazu; Tsuchiya, Shingo; Matsuura, Kiyotaka \\
\hline Citation & $\begin{array}{l}\text { Acta Materialia, 59(14), 5700-5709 } \\
\text { https://doi.org/10.1016j.actamat.2011.05.045 }\end{array}$ \\
\hline Issue Date & 2011-08 \\
\hline Doc URL & http://hdl.handle.net/2115/47106 \\
\hline Type & article(author version) \\
\hline File Information & A M59-14_5700-5709.pdf \\
\hline
\end{tabular}

Instructions for use 


\title{
Formation condition of coarse columnar austenite grain structure in peritectic carbon steels by discontinuous grain growth mechanism
}

\author{
Munekazu Ohno, Shingo Tsuchiya and Kiyotaka Matsuura
}

Division of Materials Science and Engineering, Faculty of Engineering, Hokkaido University, Kita 13 Nishi 8, Kita-ku, Sapporo, Hokkaido, 060-8628, Japan

\begin{abstract}
The discontinuous grain growth leading to coarse columnar austenite grain (CCG) structure in as-cast peritectic carbon steels is analyzed by means of two- and three-dimensional phase-field simulations. On the basis of theory of grain growth, the condition for the discontinuous grain growth to occur is elucidated in terms of cooling condition and material properties. The theoretical analysis well explains the results of the phase-field simulations and it should be useful in predicting the formation of the CCG structure in continuously-cast slabs.
\end{abstract}

Keywords; Casting; Columnar grain; Phase-field simulation; Abnormal grain growth; Peritectic solidification 


\section{Introduction}

Casting processes of peritectic carbon steels involve crystallization of primary $\delta$-ferrite, the peritectic reaction and transformation, followed by $\gamma$-austenite grain growth. Since the $\delta$ or liquid phase acts as a pinning phase for the $\gamma$ grain growth, the temperature at which the peritectic transformation completes, $T_{\gamma}$, corresponds to the temperature for the onset of $\gamma$ grain growth [1]. The as-cast microstructure in continuously-cast (CC) slabs of peritectic carbon steels consists of a coarse columnar $\gamma$ grain (CCG) structure. The CCG structure should be avoided because it causes occurrence of the surface cracking and low ductility of the slabs [1,2]. Therefore, detailed understanding of the formation kinetics of the CCG structure is one of the most important issues in the field of solidification and casting of steels.

In the experimental study on a unidirectional solidification at slow cooling rates, it was observed that the CCG develop at temperatures below $T_{\gamma}$ along the temperature gradient [3]. Importantly, fine columnar $\gamma$ grains (FCG) always exist ahead of the growing $\mathrm{CCG}$, more precisely, in the temperature region between the peritectic reaction temperature, $T_{p}$, and $T_{\gamma}$. The short-axis diameter of FCG is comparable to the primary dendrite arm spacing (DAS). Then, a model for predicting the $\gamma$ grain size in $\mathrm{CC}$ slabs was proposed with the assumption that continuous grain growth from FCG leads to the CCG structure during the cooling process below $T_{\gamma}[4,5]$. However, the formation scenario of CCG structure by the continuous grain growth from FCG has not been well substantiated experimentally or theoretically. The formation kinetics of the CCG structure remains to be clarified.

In our recent study [6], the formation process of the CCG in a metal model casting for hyperperitectic carbon steels $(0.2$ and $0.35 \mathrm{mass} \% \mathrm{C}$ steels $)$ was investigated by means of a rapid unidirectional solidification method which realizes a cooling condition quite similar to that near the slab surface in $\mathrm{CC}$ processes. It was found that the CCG structure develops along the temperature gradient and the FCG always form ahead of the developing CCG region. Of particular importance in our findings is that the continuous grain growth from FCG to CCG does not arise, but the CCG originates from the discontinuous growth process. The microstructures observed in solidifying samples are schematically illustrated in Fig. 1. In the beginning of the solidification, the columnar $\delta$ dendrites develop from the mold wall. The $\gamma$ grains appear near the mold side and grow to CCG, although this initial process was not fully elucidated. Ahead of the CCG, as shown in Fig. 1(a), new $\gamma$ grains form in $\delta$ dendrites below $T_{p}$ and they grow to FCG. In the FCG region, the liquid phase plays a role of a "pin" to prevent the growth of FCG along its short-axis direction, thus making the short-axis diameter of the 
FCG comparable to the primary DAS of $\delta$ dendrites. The liquid phase in the FCG region also retards the vertical motion of the FCG/CCG region boundary (FCRB) shown in Fig. 1. As the cooling proceeds, the position of $T_{\gamma}$ moves away from the mold side and the liquid phase accordingly vanishes in that region. The FCRB then moves upward with the growth of CCG and the shrink of FCG near FCRB, as shown in Fig. 1(b). At a fixed position of the cast sample, therefore, the grain size discontinuously increases by about one order of magnitude from FCG to CCG when the temperature falls below $T_{\gamma}$. This process corresponds to the discontinuous grain growth and is in marked contrast to the continuous grain growth assumed in Refs. [4, 5].

As described above, our recent study revealed that the CCG formation is actually ascribable to the discontinuous grain growth. However, its kinetics has not been well comprehended yet. It is especially imperative for the prevention of CCG formation to quantitatively ascertain a critical condition for the occurrence of the discontinuous grain growth. In the present study, the occurrence of the discontinuous grain growth in various cooling conditions and the CCG formation by its mechanism are first verified by means of two- and three-dimensional phase-field simulations. On the basis of a theory of grain growth, subsequently, we find out the formation condition of the CCG structure in terms of cooling conditions, initial grain size and grain boundary mobility. It will be demonstrated that the formation condition of the CCG put forward in this study is quite consistent with the results of the phase-field simulations and it can be utilized for the prediction of the CCG formation in the practical CC slabs.

\section{Calculation method}

\subsection{Phase-field model}

The discontinuous grain growth for the CCG formation illustrated in Fig. 1 essentially involves several phenomena characterized in different spatial and time scales such as the columnar dendrite growth, the peritectic reaction/transformation and the grain growth. The accurate description of the whole process requires extensive computational resources, which are impractical. In the present study, we disregard the details on the formation of FCG structure for simplicity. The pinning pressure of the liquid is furthermore taken into account within an approach of the effective grain boundary mobility as in Ref. [7]. Our main concern is thus directed to the $\gamma$ grain growth from FCG under a temperature gradient.

In this study, we utilized the multi-phase-field model proposed by Steinbach and Pezzola [8] and modified by Kim et al. [9]. In what follows, we provide only essential formulae necessary for the present study. Within the phase-field model, the 
grain structure is characterized by a set of phase-fields, $\left\{\phi_{i}(\mathbf{r}, t)\right\}$. The phase-field, $\phi_{i}(\mathbf{r}, t)$, describes a probability of finding a grain having a crystallographic orientation, $i,(i=1,2$, $3, \cdots, N)$. Here, $\mathbf{r}$ is the spatial coordinate, $t$ is the time and $N$ is the total number of the orientations counted in the simulation. $\phi_{i}$ is allowed to vary from 0 to 1 . The normalization condition, $\sum_{i}^{N} \phi_{i}(\mathbf{r}, t)=1$, is held in each spatial point. The grain boundary is described as the diffuse interface in which the orientation of grains gradually changes. The time evolution of $\phi_{i}$ is described by the following equation [9],

$$
\frac{\partial \phi_{i}}{\partial t}=-\frac{2 L}{n} \sum_{j \neq i}^{N} s_{i} s_{j}\left(\frac{\delta F}{\delta \phi_{i}}-\frac{\delta F}{\delta \phi_{j}}\right) .
$$

where $L$ is the phase-field mobility, $s_{i}$ is a step function with $s_{i}=1$ for $\phi_{i}>0$ and $s_{i}=0$ otherwise and $n$ represents the number of phases existing in a given spatial point as defined by $n=\sum_{i}^{N} s_{i}(\mathbf{r}, t)$. The functional derivative on the right-hand side of eq.(1) is given as,

$$
\frac{\delta F}{\delta \phi_{i}}=\sum_{k \neq i}^{N}\left(\frac{\varepsilon^{2}}{2} \nabla^{2} \phi_{k}+\omega \phi_{k}\right),
$$

where $\varepsilon$ is the gradient energy coefficient and $\omega$ represents a height of the parabolic potential with a double obstacle in the expression of the free energy functional, $F$. These parameters are related to the interface thickness, $W$, and the grain boundary energy, $\sigma$, as given by $\varepsilon=2 \sqrt{W \sigma} / \pi$ and $\omega=4 \sigma / W$ [9]. Hence, when the values of $\sigma$ and $W$ are given, $\varepsilon$ and $\omega$ are uniquely fixed. We do not take into consideration the crystallographic anisotropies of the grain boundary energy and mobility in this study.

It is quite important to note that the Allen-Cahn type equation (1) exactly reproduces the following well-known relationship for the curvature-driven motion of boundary [9],

$$
v=-m \sigma \kappa,
$$

where $v$ is the migration velocity of the grain boundary, $m$ is the grain boundary mobility and $\kappa$ is the mean curvature of the grain boundary. The phase-field mobility, $L$, is associated with $m$ as follows,

$$
L=m \sigma / \varepsilon^{2} \text {. }
$$

Therefore, the kinetics of the grain growth is entirely controlled by the parameter $L$ in the phase-field simulation. We describe the temperature dependence of $L$ by 
$L(T)=a_{0} \cdot \exp (-Q /(R T))$. Here, $R$ is the gas constant and the parameters $a_{0}$ and $Q$ can be determined based on the experimental data.

In order to simulate coalescence-free grain growth within reasonable computational time, we employed the active parameter tracking (APT) algorithm [9-11]. In the APT algorithm, only the phase-fields which are non-zero and are regarded as "active" in a given point and its nearest neighbor points are stored (updated) and solved. Since the total number of the active phase-fields in each spatial point is always much less than $N$, the increase of $N$ does not incur the increase in the computational burden. More details on the APT algorithm can be found in Refs. [9-11]. We used the APT algorithm proposed by Kim et al.[9].

\subsection{Computational system and parameters}

The parameters used in the present study are summarized in Table 1. The two-dimensional (2D) simulations were carried out on $1000 \times 500$ square grid points for $x$ and $y$-axes, respectively. The periodic boundary condition was set along $x$-direction. Since the temperature gradient was unidirectionally imposed along $y$ direction, zero flux boundary condition for $\phi_{i}$ was applied to the upper and lower edges in $y$-direction.

We focused on several initial structures (FCG structure) characterized by the initial short-axis diameter, $d_{\text {initial }}$, and initial aspect ratio, $a_{\text {initial }}$, as listed in Table 1 . Here, $d_{\text {initial }}$ is the average grain diameter along $x$-axis estimated by the linear intercept method. The aspect ratio is defined as the ratio of the grain diameter of $y$-axis to that of $x$-axis. The initial equiaxed grain structures of $a_{\text {initial }}=1$ were obtained by solving eq.(1) for different annealing periods at $\mathrm{T}=1743 \mathrm{~K}$ starting from randomly distributed grains. As for the initial columnar grain structure of $a_{\text {initial }}=3$, large values were assigned to the grid spacing of $y$-axis in the preliminary simulations to produce columnar grains elongated along $y$-direction and the structures were finally annealed on the square grid for a certain period. We reassigned a different orientation number, $i$, to each grain in the initial structures thus prepared, with the aim of simulating the coalescence-free grain growth in the cooling process. As an example, the initial structure of $d_{\text {initial }}=150 \mu \mathrm{m}$ and $a_{\text {initial }}=3$ is shown in Fig. 2(a).

The $\gamma$ grain growth in cast steels has been discussed in the light of the temperature gradient, $G$, and the cooling rate, $\dot{T}$, in early studies $[1,3-5,14]$. In view of this, we use $G$ and $\dot{T}$ to specify the cooling process. For simplicity, $G$ and $\dot{T}$ are assumed to be constant with time and space, unless otherwise stated. Therefore, the position of $T_{\gamma}$ moves at a constant velocity, $V_{T}=\dot{T} / G$, which is called the thermal velocity in this paper. The ranges of $G$ and $\dot{T}$ focused in this study are shown in Table 
1.

We found in the previous study [6] that the short-axis diameter of FCG is always comparable to the primary DAS during the CCG formation process. This implies quite a strong pinning effect of the liquid at temperatures above $T_{\gamma}$. In the present simulation, hence, the grains are not allowed to grow at temperatures above $T_{\gamma}$ by assigning null value to $L$, which is regarded as a special case of the effective mobility approach [7]. In other words, the existence of liquid is implicitly described by zero mobility of grain boundary. When the temperature decreases down to $T_{\gamma}, L$ takes a finite value depending on the local temperature, which indicates that the liquid phase vanishes in that region. In the beginning of all the simulations, the temperature was set to be higher than $T_{\gamma}$ over the whole system. The lower edge in $y$-direction corresponds to the lowest temperature part, thereby the mold side. The simulation was stopped when the temperature at the upper edge of $y$-axis reaches $T_{\gamma}$.

In order to confirm the validity of the discussions, we conducted the three-dimensional (3D) simulation, using the system consisting of $270 \times 480 \times 270$ gird points for $x, y$, and $z$ axes. The simulation conditions are the same as those in $2 \mathrm{D}$ simulation. The periodic boundary condition was set along $x$ - and $z$-directions, while the zero flux boundary condition for $\phi_{i}$ was applied to both the edges in $y$-direction. The linear temperature gradient, $G$, is imposed along $y$-direction. The initial structure consists of equiaxed grains with the average equivalent volume diameter of $200 \mu \mathrm{m}$ in the case of 3D simulation.

It should be pointed out that the above-mentioned process of our concern bears a resemblance to a directional annealing process of polycrystalline materials. Development of columnar grain structures during the directional annealing has been investigated by means of Monte Carlo simulation [15] and front-tracking model [16-18] and, in fact, one can find a certain similarity between the present and their results. As might be expected, however, the findings in these studies are not entirely applicable to the present case, mainly because of the difference in the cooling process. In particular, one fails to grasp an understanding for the present results based on relations defining a critical condition for the occurrence of the discontinuous growth proposed in the early studies $[16,17]$.

\section{Results and discussion}

\subsection{Microstructural evolutions for continuous and discontinuous grain growth}

Figures 2(b) demonstrates the microstructural evolution process calculated in $2 \mathrm{D}$ system for $T_{\gamma}=1743 \mathrm{~K}, \dot{T}=1.0 \mathrm{~K} / \mathrm{s}$ and $G=6.0 \times 10^{3} \mathrm{~K} / \mathrm{m}$. The initial structure is the 
one shown in Fig. 2(a). The bottom of each snapshot is the mold side. The temperature is denoted on the left-hand axis in each snapshot and the arrow on the right-hand side of the snapshot indicates the positions of $T_{\gamma}$. The grain growth continuously takes place from FCG in local regions after the local temperatures decrease down to $T_{\gamma}$. The grain size continuously changes near the position of $T_{\gamma}$. This process is classified into the continuous grain growth. The aspect ratio in the final structure, $a_{f}$, is about 1.7 which is lower than $a_{\text {initial }}$. Hence, the continuous process leads to a relatively equiaxed grain structure.

Figure 2(c) shows the microstructural process calculated for a larger temperature gradient, $G=1.4 \times 10^{4} \mathrm{~K} / \mathrm{m}$. The other calculation conditions are the same as in Fig. 2(b). One can readily notice a striking difference between Figs. 2(b) and (c). The structure at $t=40 \mathrm{~s}$ is composed of well-distinguishable grain assemblies of FCG (upper) and CCG (lower part). The FCG discontinuously changes into CCG in the vicinity of $T_{\gamma}$ and the FCRB is conspicuously identified. The grain growth proceeds by the vertical motion of the FCRB in line with the migration of $T_{\gamma}$ position. This is exactly the discontinuous grain growth found in our previous study [6]. The final structure consists of fully columnar grains with $a_{f}$ as high as 6.2. It was shown in our simulations that $a_{f}$ becomes higher than $a_{\text {initial }}$ only when the discontinuous growth occurs.

\subsection{Condition for occurrence of discontinuous grain growth}

As mentioned above, the continuous and discontinuous processes, respectively, result in lower and higher values of $a_{f}$ than $a_{\text {initial }}$. In Fig. 3, $a_{f}$ is plotted with respect to $V_{T}$. In this figure, $\dot{T}$ is fixed in each set of data. The increase in $V_{T}$ corresponds to the decrease in $G$ because $V_{T}$ was defined as $\dot{T} / G$. The horizontal dotted line denotes $a_{\text {initial }}$. In all the cases, $a_{f}$ is nearly equal to $a_{\text {initial }}$ in high $V_{T}$ region. This is because the grain growth takes place only for a short period of time when $V_{T}$ is high. At $\dot{T}=0.1$ and 1.0 $\mathrm{K} / \mathrm{s}$, $a_{f}$ gradually decreases with decrease in $V_{T}$ until about $V_{T}=1 \times 10^{-4} \mathrm{~m} / \mathrm{s}$. This is because the grains are allowed to grow for a longer period of time in the lower $V_{T}$ process and the grains thereby become more equiaxed shape. However, when $V_{T}$ falls below $1 \times 10^{-4} \mathrm{~m} / \mathrm{s}, a_{f}$ drastically increases as $V_{T}$ decreases. That is, the discontinuous grain growth emerges. The drastic increase of $a_{f}$, i.e., the discontinuous growth also appears at $\dot{T}=10$ and $20 \mathrm{~K} / \mathrm{s}$. In these cases, however, $a_{f}$ starts to decrease with further decrease of $V_{T}$. From the microstructural change, it was found that both the discontinuous and continuous grain growth hardly occur in such low $V_{T}$ regions. The reason for this will be explained later. As $\dot{T}$ increases, the maximum value of $a_{f}$ decreases and, when $\dot{T}=50 \mathrm{~K} / \mathrm{s}, a_{f}$ is always less than 3 , which indicates that no 
discontinuous grain growth arises in this condition. It should be pointed out that our focus in this paper is placed entirely on the elucidation of the critical condition for the occurrence of the discontinuous grain growth. Hence, we omit the detail on the grain size in each condition to make our discussion concise. The detail on the grain size will be discussed elsewhere [19].

The results for $T_{\gamma}=1743 \mathrm{~K}, d_{\text {initial }}=150 \mu \mathrm{m}$ and $a_{\text {initial }}=3$ are summarized in Fig. 4 . The different types of symbols distinguish the range of $a_{f}$. The solid line is the calculated result of eq.(7) which will be expounded upon later. This figure represents the structure map specifying the shape of grains (equiaxed or columnar) formed in the cooling process. It is seen that the discontinuous grain growth, i.e., the CCG formation arises in a certain range of the cooling conditions. In what follows, we discuss what determines the occurrence of the discontinuous grain growth based on the theory of grain growth $[20,21]$ and find out a condition for the discontinuous grain growth to occur in terms of the cooling conditions, initial structure and grain boundary mobility.

There are several theoretical and computational studies on the discontinuous grain growth [20-25]. Humphreys proposed a theory for the occurrence of the discontinuous growth in which a "particular" grain preferentially grows in an assembly of grains due to the difference in boundary energy and mobility between the particular grain and assembly [21]. One of his important findings is that in the system with the isotropic grain boundary energy, the discontinuous growth of the particular grain takes place only when the grain boundary mobility of the particular grain is higher than that of assembly. In the process of our focus, the mobility in FCG region is zero as long as the pinning effect exists. However, when the pinning effect vanishes, the mobility in FCG region is always higher than that in CCG region, because of the higher temperature in FCG region due to the temperature gradient. Then, it should be reasonable to postulate that the discontinuous grain growth of our concern occurs when $V_{T} \leq V_{\gamma}$ is

satisfied, where $V_{\gamma}$ represents the migration velocity of FCRB. When $V_{T} \leq V_{\gamma}$ is held, the motion of FCRB can always follow the migration of $T_{\gamma}$ position. Then, the coarsening of FCG does not take place at all due to the pinning effect. When $V_{T}>V_{\gamma}$, however, the pinning effect vanishes in the FCG region prior to the FCRB migration and the grain boundary mobility in FCG becomes higher than that in CCG. As speculated from the Humphreys' theory, then, the preferential growth of CCG is prohibited. Therefore, the relation of $V_{T} \leq V_{\gamma}$ should represent the condition for the 
discontinuous grain growth to occur.

Our concern is now directed to the precise calculation of $V_{\gamma}$. According to Humpherys' theory, the growth rate of the particular grain depends on the ratio between the grain sizes of the particular grain and assembly[21]. Therefore, it seems to be reasonable to consider that $V_{\gamma}$ depends on the ratio between CCG and FCG sizes. Then, one may consider that $V_{\gamma}$ should be described with distinction between initiation and continuation of the discontinuous growth, because the ratio between CCG and FCG sizes is time dependent. In the following discussion, hence, we denote the initial and steady state velocities of FCRB by $V_{\gamma}^{i}$ and $V_{\gamma}^{c}$, respectively. It should be pointed out that a rough estimation of $V_{\gamma}^{c}$ was already attempted in our previous study [6] with the assumption that CCG is infinitely large. However, we found it indispensable for a proper estimation of $V_{\gamma}^{c}$ as well as $V_{\gamma}^{i}$ to presuppose an appropriate shape of FCRB (obeying Young's law) and to base the theory of grain growth underlying a correct expression for the grain boundary mobility and also to take the effect of the temperature gradient into account in the mobility of FCRB.

Hillert developed the theory of grain growth on the basis of the Lifshitz and Slyozov theory [20]. The Hillert theory is predicated on the following equation for the time evolution of the grains with a radius, $r$,

$$
\begin{aligned}
\frac{d r}{d t}= & \alpha m \sigma\left(\frac{1}{r_{c}}-\frac{1}{r}\right) \\
& =M\left(\frac{1}{r_{c}}-\frac{1}{r}\right),
\end{aligned}
$$

where $\alpha$ is a constant depending on the dimensionality of the system and shape of the grains, $m$ and $\sigma$ are the grain boundary mobility and energy, respectively, as already defined in eq.(3). $r_{c}$ is a critical radius below which the grain of a radius shrinks. $r_{c}$ is related to the average grain radius, $\left\langle r>\right.$, as $r_{c}=g<r>$ with $g=1$ for $2 \mathrm{D}$ and $9 / 8$ for $3 \mathrm{D}$. The time evolution of $r_{c}$ is given as,

$$
\frac{d r_{c}^{2}}{d t}=\frac{1}{2} M
$$

This theory is now applied to the CCG formation process as detailed below. 
The grain structure in the vicinity of the FCRB is schematically depicted in Fig. 5. It is first noticed that there are the triple junctions formed by the grain boundaries of CCG and FCG (drawn by the dashed lines). As the short-axis diameter of the CCG decreases, the number of this type of triple junction increases. Here it should be mentioned that the present phase-field model exactly reproduces von Neumann-Mullins law of 2D grain growth kinetics [9]. This indicates the fact that the triple junctions do not affect the grain growth kinetics and the contact angles at triple junctions always comply with the equilibrium relationship. In order to ensure the consistency with this fact, we assume no influence of the triple junctions on the kinetics here, which is actually a common practice in the theories of grain growth. Hence, the triple junctions denoted by the dashed lines do not affect the motion of FCRB. They move upward merely to follow the migration of FCRB. Therefore, the grain diameter of the CCG is irrelevant to the kinetics of the FCRB motion. Then, the grain boundary denoted by the bold line entirely determines the migration velocity of FCRB. The local curvature radius of this segment is denoted by $r^{\prime}$. The migration velocity of this segment corresponds to the shrinking velocity of the grain of radius $r^{\prime}$ in an infinitely large grain, i.e., $r_{c} \approx \infty$ in eq.(5). Then, $V_{\gamma}^{i}$ is given as $V_{\gamma}^{i}=-d r^{\prime} / d t=M / r^{\prime}$. Here, $r^{\prime}$ is associated with $d_{\text {initial }}$ as written by $r^{\prime}=\xi d_{\text {initial }}$. Here $\xi$ is given as $\xi=\left(1+\tan ^{2} \theta\right)^{1 / 2} /(2 \tan \theta)$ with the angle, $\theta$, defined in Fig. 5. Since the contact angle at the triple junction is $120^{\circ}$, one finds $\tan \theta=1 / \sqrt{3}, \xi=1$ and thereby $r^{\prime}=d_{\text {initial }}$. Then, $V_{\gamma}^{i}$ is rewritten as $V_{\gamma}^{i}=M / d_{\text {initial. }}$. It is emphasized here that $d_{\text {initial }}$ is kept to be a constant due to a strong pinning effect. In addition, the velocity of FCRB is independent of the size of CCG. Namely, there is no time-dependent quantity involved in the expression of $V_{\gamma}^{i}$. Therefore, $V_{\gamma}^{i}$ is virtually equivalent to $V_{\gamma}^{c}$. Hereafter, hence, we will discuss the migration velocity of the FCRB without the distinction between the initiation and continuation and describe it by $V_{\gamma}$.

The effect of the temperature gradient on FCRB motion needs to be considered. The temperature dependence of $M$ can be expressed as $M(T)=M_{0} \exp \left(-Q /\left(R T_{\gamma}^{*}\right)\right)$ with a coefficient $M_{0}$ and the temperature at the FCRB, $T_{\gamma}{ }^{*}$. Generally, $T_{\gamma}{ }^{*}$ is nearly equal to $T_{\gamma}$. When the temperature gradient is large, however, it should deviate from $T_{\gamma}$. In Fig. 
5 , the position of $T_{\gamma}$ should be located high above the FCRB in order that the shape of FCRB obeys the Young's law. Meanwhile, it should not be too high to avoid FCG from growing. If the temperature at the center of circle of curvature is approximated to be $T_{\gamma}$, the temperature at the rearmost point of FCRB is given by $T_{\gamma}-G \cdot r^{\prime}$ which controls the migration velocity of FCRB. Hence, $V_{\gamma}$ is finally given in $2 \mathrm{D}$ system as,

$$
V_{\gamma}=M_{0} \exp \left(-\frac{Q}{R\left(T_{\gamma}-G \xi d_{\text {initial }}\right)}\right) \frac{1}{\xi d_{\text {initial }}},
$$

where $\xi$ is given to be 1 for $2 \mathrm{D}$ system. For the calculation of eq.(7), we estimated $M_{0}$ $=3.0 \times 10^{-3} \mathrm{~m}^{2} / \mathrm{s}$ from simulations of isothermal grain growth. As discussed above, the discontinuous grain growth occurs when $V_{T} \leq V_{\gamma}$ is satisfied. Importantly, $V_{\gamma}$ given by eq.(7) does not depend on the size of CCG and, therefore, the condition $V_{T} \leq V_{\gamma}$ exactly represents the critical condition for both the initiation and continuation of the discontinuous grain growth.

The relation between $\dot{T}$ and $G$ at $V_{T}=V_{\gamma}$ was calculated from the relation, $V_{T}=\dot{T} / G$, and eq.(7) and is represented by the solid line in Fig. 4. One can see that the relation of $V_{T}=V_{\gamma}$ is fully consistent with the results of the phase-field simulations. In the range of $\dot{T}<0.1$ and $G<10^{3}$, the critical value of $G$ is almost proportional to $\dot{T}$ according to $\dot{T} / G=M\left(T_{\gamma}\right) / d_{\text {initial }}$. On the other hand, the proportionality does not hold true in the range of $G=10^{3}-10^{6}$, because $V_{\gamma}$ decreases as $G$ increases according to eq.(7). When $G$ is very large, the discontinuous grain growth hardly occurs due to small value of $V_{\gamma}$, resulting in $a_{f}$ close to $a_{\text {initial }}$ as is consistent with the simulation results (triangle symbols). Figure 6 shows the results for different values of $d_{\text {initial }}$ and $T_{\gamma}$. The agreement between the proposed relation and the simulation results is excellent in both the cases. Furthermore, we confirmed that the relation of $V_{T}=V_{\gamma}$ calculated by eq.(7) well explains the other cases for different set of $d_{\text {initial }}$ and $T_{\gamma}$, although not shown here. From the comparison between Figs. 4 and 6, it is evident that small $d_{\text {initial }}$ and/or high $T_{\gamma}$ results in a wide range of the discontinuous growth region, in other words, a strong tendency for the CCG formation. In fact, our recent experimental study on rapid unidirectional solidification of a hyperperitectic carbon steel demonstrated that the as-cast $\gamma$ grain structure changes from CCG to equiaxed grain structure due to the reduction of $T_{\gamma}$ associated with $\mathrm{Cr}$ addition [26].

In eq.(7), $V_{\gamma}$ is entirely determined by the local curvature of the FCG and is not related to $a_{\text {initial }}$. Therefore, the difference in $a_{\text {initial }}$ should not alter the structure map, 
which was, in fact, confirmed by comparing the simulation results of $a_{\text {initial }}=1$ and 3 . The different value of $a_{\text {initial }}$ actually yields the difference in the final grain size but not in the critical condition for the CCG formation. This point will be discussed elsewhere [19].

\subsection{Transition between continuous and discontinuous grain growth processes}

As explained above, the condition $V_{T} \leq V_{\gamma}$ gives rise to the discontinuous grain growth. Although our discussion has been limited to the processes for constant $G$ and $\dot{T}$, the present structure map should be utilized for more general cases, i.e., cooling processes involving temporal and spatial variations of $G$ and $\dot{T}$. Figures 7 (a) and (b) show the initial grain structure characterized by $d_{\text {initial }}=150 \mu \mathrm{m}$ and $a_{\text {initial }}=1$ and the final grain structure after cooling process, respectively. $T_{\gamma}$ was set to be $1743 \mathrm{~K}$. The structure map for this condition corresponds to Fig. 4. In this cooling process, $G$ is kept to be $2.0 \times 10^{5} \mathrm{~K} / \mathrm{m}$, while $\dot{T}$ monotonically decreases with time from 20 to $5 \mathrm{~K} / \mathrm{s}$. The cooling condition at $T_{\gamma}$ position is initially in the continuous growth region of the structure map and it moves to the discontinuous growth region in Fig. 4. The arrow in Fig. 7(b) indicates the position where $V_{T}=V_{\gamma}$ is satisfied. As expected, the grain structure changes from the equiaxed type to columnar type near the position for $V_{T}=V_{\gamma}$. Figure 7 (c) shows the structure after the cooling process in which $G$ was spatially varied from $1.6 \times 10^{4} \mathrm{~K} / \mathrm{m}$ at the bottom edge to $2.0 \times 10^{2} \mathrm{~K} / \mathrm{m}$ at the top edge. The spatial variation of $G$ was given by a quadratic function. $\dot{T}$ is fixed to be $1.0 \mathrm{~K} / \mathrm{s}$. The arrow indicates the position for $V_{T}=V_{\gamma}$. As is consistent with Fig. 4, the grain structure changes from the columnar type to equiaxed type near the position for $V_{T}=V_{\gamma}$. Hence, the structure map can be utilized for nonsteady cooling processes. When $G-\dot{T}$ relation at $T_{\gamma}$ is known, the time and position at which the CCG form in CC slabs can be predicted based on the structure map.

\subsection{Three-dimensional simulations}

In order to verify the present discussion in more realistic case, we performed $3 \mathrm{D}$ simulations. Figure 8 shows the results for $T_{\gamma}=1743 \mathrm{~K}, d_{\text {initial }}=200 \mu \mathrm{m}, a_{\text {initial }}=1$ and $\dot{T}=10 \mathrm{~K} / \mathrm{s}$. The grain growth under $G=1.0 \times 10^{4} \mathrm{~K} / \mathrm{s}$ is shown in Fig. 8(a). It proceeds in a continuous manner. However, the grain growth under a larger value of $G$ in Fig. 8(b) is the discontinuous process which results in the CCG structure elongated along $y$-direction. The results of 3D simulations are summarized in Fig. 9.

The validity of the relation $V_{T} \leq V_{\gamma}$ for $3 \mathrm{D}$ system is discussed. The pre-exponential factor $M_{0}$ in eq.(7) was first estimated in 3D system. It was shown in 
$3 \mathrm{D}$ isothermal simulation that it is roughly twice as large as the value of $2 \mathrm{D}$, which is fairly consistent with the Hillert theory [20]. The factor $\xi$ in eq.(7) deviates from 1.0 in $3 \mathrm{D}$, because the shape of FCRB is different from the one in $2 \mathrm{D}$ system. The estimation for the mean curvature of FCRB surface in 3D system requires the quadruple junction point as well as the triple junction line to be considered. For this, we assume that the cross-sectional shape of FCG on $x-z$ plane perpendicular to the moving direction of FCRB (y-direction) is a regular hexagon. We denote the short and long diagonals of the hexagon by $d_{1}$ and $d_{2}$, respectively. Then, the mean curvature of FCRB surface, $r^{\prime}$, is expressed as $r^{\prime}=\left(\xi_{1} d_{1}+\xi_{2} d_{2}\right) / 2$. As is similar to $2 \mathrm{D}$ case, $\xi_{i}$ is given by $\xi_{i}=\left(1+\tan ^{2} \theta_{i}\right)^{1 / 2} /\left(2 \tan \theta_{i}\right)$. Here, $\theta_{1}\left(\theta_{2}\right)$ is the angle between $y$-axis and a vector normal to the FCRB surface at the midpoint of a side (at a vertex of the hexagon) which corresponds to the triple junction (quadruple junction). Based on the Young's law, one can find $\tan \theta_{1}=1 / \sqrt{3}$ and approximate $\tan \theta_{2} \approx 1 / \sqrt{2}$. Then, $\xi$ is estimated to be about 0.78. As is naturally expected, therefore, $\xi d_{\text {initial }}$ is slightly larger than the equivalent volume radius of FCG. The calculated relation for $V_{T}=V_{\gamma}$ is indicated by the solid line in Fig. 9. It can be seen that the proposed relation well explains the results of the $3 \mathrm{D}$ simulations. The critical condition we put forward in this study is thus validated for $3 \mathrm{D}$ systems of practical importance.

\section{Conclusions}

Our recent experiments demonstrated that the formation of as-cast coarse columnar $\gamma$ grains (CCG) in peritectic carbon steels is ascribable to the discontinuous grain growth process [6]. In this study, the occurrence of discontinuous grain growth in the various cooling conditions and the CCG formation by its mechanism were verified by means of two- and three-dimensional phase-field simulations. Based on the theory of grain growth, furthermore, we developed the relation describing the critical condition for the discontinuous grain growth to start and continue, i.e., the formation condition of CCG structure. The structure map constructed in this analysis should be useful in predicting the CCG formation in continuously-cast slabs. Since our discussion in this paper was centered on the elucidation of the critical condition, we did not detail the $\gamma$ grain size in each condition. It was found in 2D system, however, that the coarsest grain structure emerges by the discontinuous growth in the vicinity of the critical condition. This will be discussed elsewhere [19].

In reality, the short-axis diameter of fine columnar $\gamma$ grains (FCG), $d_{\text {initial }}$, corresponds to the primary DAS, $\lambda_{1}$. Hence, although we used the constant values of 
$d_{\text {initial }}$ irrespective of the cooling conditions, the practical application of the present relationship needs the consideration of the dependence of $d_{\text {initial }}$, i.e., $\lambda_{1}$ on $G$ and $\dot{T}$. Moreover, we did not take into account the orientational dependences of the grain boundary energy and mobility and the effect of the motions of the triple and quadruple junctions $[27,28]$. These remains to be addressed in the future work to make an important progress in understanding and controlling the formation of as-cast coarse columnar $\gamma$ grain structure.

\section{Acknowledgments}

This work is supported by $18^{\text {th }}$ ISIJ Research Promotion Grant and Grant-in-Aid for Young Scientists (A) (No. 22686067) from MEXT, Japan. Also, the author M.O. acknowledges partial financial support from the Next Generation Super Computing Project, Nano-science Program, MEXT, Japan. 


\section{References}

[1] Maehara Y, Yasumoto K, Sugitani Y, Gunji K. ISIJ Int 1985;25:1045.

[2] Schmidt L, Josefsson Å. Scand J Metall 1974;3:193.

[3] Maruyama T, Matsuura K, Kudoh M, Itoh Y. Tetsu-to-Hagané 1999;85:585.

[4] Yoshida N, Umezawa O, Nagai K. ISIJ Int 2004;44:547.

[5] Kim HS, Kobayashi Y, Nagai K. Acta Mater 2006;54:2441.

[6] Tsuchiya S, Ohno M, Matsuura K, Isobe K. Acta Mater 2011;59:3334.

[7] Apel M, Böttger B, Rudnizki J, Schaffnit P, Steinbach I. ISIJ Int 2009;49:1024.

[8] Steinbach I, Pezzola F. Physica D 1999;134:385.

[9] Kim SG, Kim DI, Kim WT, Park YB. Phys Rev E 2006;74:061605.

[10] Vedantam S, Patnaik BSV. Phys Rev E 2006;73:016703.

[11] Gruber J, Ma N, Wang Y, Rollett AD, Rohrer GS. Model Simul Mater Sci Eng 2006;14:1189.

[12] Hondros ED. Proc R Soc (London) A 1965;A286:479.

[13] Bernhard C, Reiter J, Presslinger H. Metall Mater Trans B 2008;39B:885.

[14] Matsuura K, Itoh Y, Matsubara K. Tetsu-to-Hagané 1990;76:714.

[15] Godrey AW, Martin JW. Philos Mag A 1995;27:737.

[16] Holm EA, Zacharopoulos, Srolovitz DJ. Acta Mater 1998;46:953.

[17] Badmos AY, Frost HJ, Baker I. Acta Mater 2002;50:3347.

[18] Badmos AY, Frost HJ, Baker I. Acta Mater 2003;51:2755.

[19] Ohno M, Matsuura K. in preparation.

[20] Hillert M. Acta Metall 1965;13:227.

[21] Humphreys FJ. Acta Mater 1997;45:4231.

[22] Thompson CV, Frost HJ, Spaepen F. Acta Metall 1987;35:887.

[23] Rollett AD, Srolovitz DJ, Anderson MP. Acta Metall 1989;37:1227.

[24] Rollett AD, Mullins WW. Scripta Mater 1997;36:975.

[25] Grest GS, Anderson MP, Srolovitz DJ, Rollett AD. Scripta Mater 1990;24:661.

[26] Tsuchiya S, Ohno M, Matsuura K, Isobe K. ISIJ Int 2010;12:1959.

[27] Gottstein G, Ma Y, Shvindlerman LS. Acta Mater 2005;53:1535.

[28] Barrales Mora LA, Mohles V, Shvindlerman LS, Gottstein G. Acta Mater 2008;56:1151. 
Table 1 Simulation parameters used in this study

\begin{tabular}{l|l}
\hline \multicolumn{1}{c|}{ Parameters } & \multicolumn{1}{c}{ Values } \\
\hline \hline Grid spacing, $\Delta x(\mathrm{~m})$ & $10^{-6}$ \\
\hline Interface thickness, $W(\mathrm{~m})$ & $6 \cdot \Delta x$ \\
\hline Grain boundary energy, $\sigma\left(\mathrm{Jm}^{-2}\right)$ & $0.79[12]$ \\
\hline Time step, $\Delta t(\mathrm{~s})$ & $\Delta x^{2} /\left(\beta L \varepsilon^{2}\right)^{*}$ \\
\hline Pre-exponential factor of phase-field mobility, $a_{0},\left(\mathrm{~m}^{3} \mathrm{~J}^{-1} \mathrm{~s}^{-1}\right)$ & $104^{* *}[13]$ \\
\hline Activation energy of phase-field mobility, $Q(\mathrm{~kJ} / \mathrm{mol})$ & $177^{* *}[13]$ \\
\hline Initial short-axis diameter, $d_{\text {initial }}\left(10^{-6} \mathrm{~m}\right)$ & $100,150,200$ \\
\hline Initial aspect ratio, $a_{\text {initial }}$ & 1,3 \\
\hline Temperature for the completion of peritectic transformation, $T_{\gamma}(\mathrm{K})$ & $1743,1643^{* *}$ \\
\hline Temperature gradient, $G(\mathrm{~K} / \mathrm{m})$ & $2 \times 10^{2}-5 \times 10^{6}$ \\
\hline Cooling rate, $\dot{T}(\mathrm{~K} / \mathrm{s})$ & $0.1-50$ \\
\hline
\end{tabular}

$* \beta$ is given to be 5 and 7 for $2 \mathrm{D}$ and $3 \mathrm{D}$ simulations, respectively.

** We do not focus on a specific steel composition in this study and hence, these values are chosen as typical values in steels. 
(a)

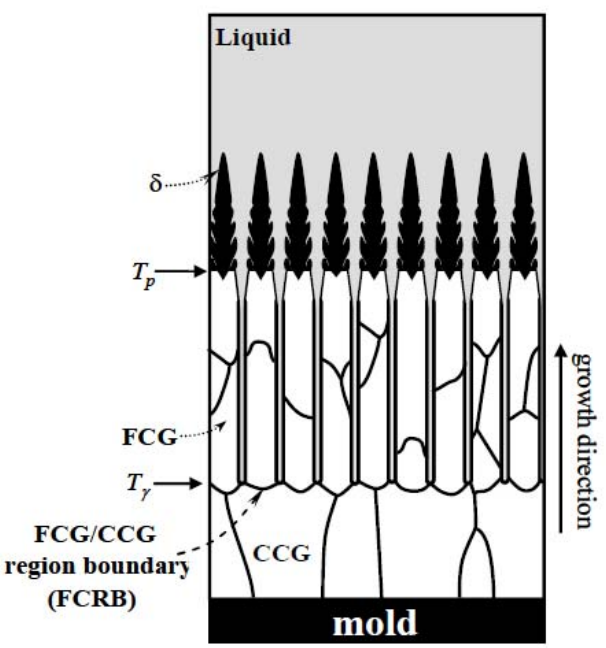

(b)

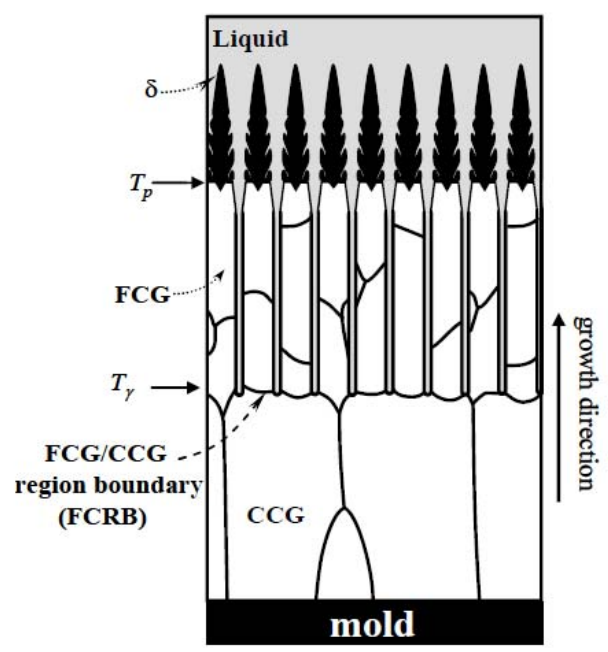

Fig. 1. Schematic illustration of CCG formation process by the discontinuous grain growth mechanism. The microstructure evolves from (a) to (b). 


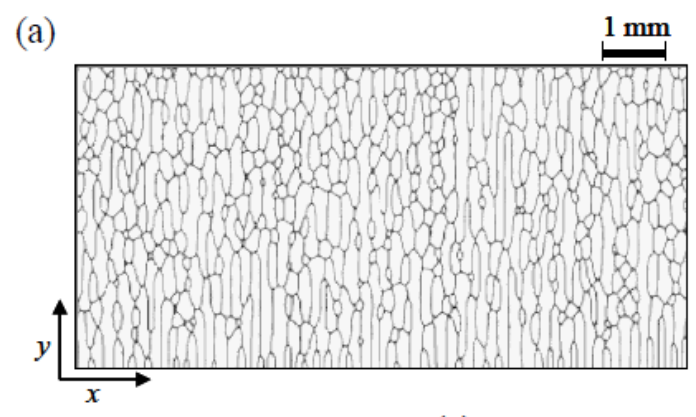

(b)

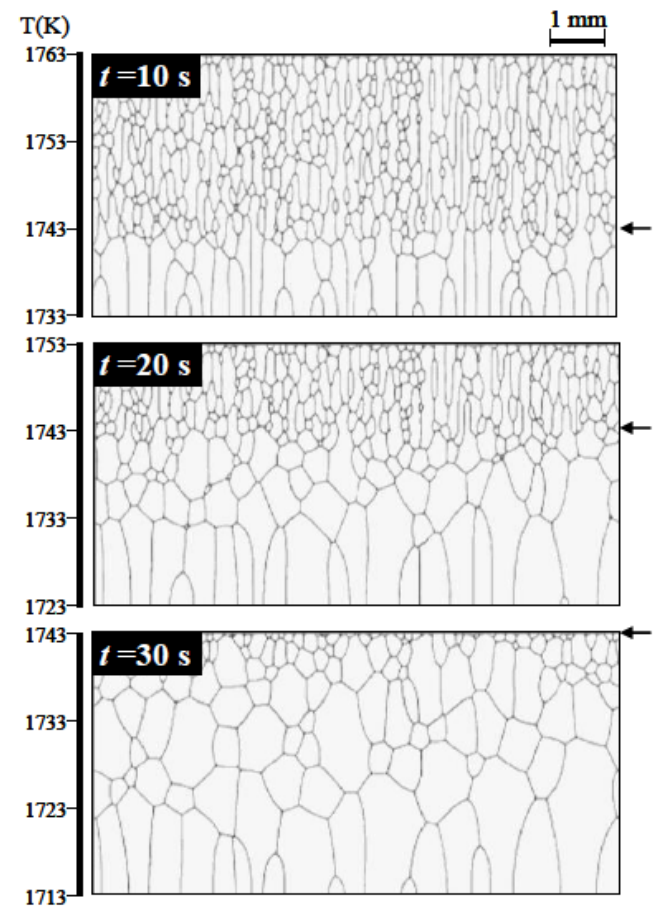

(c)

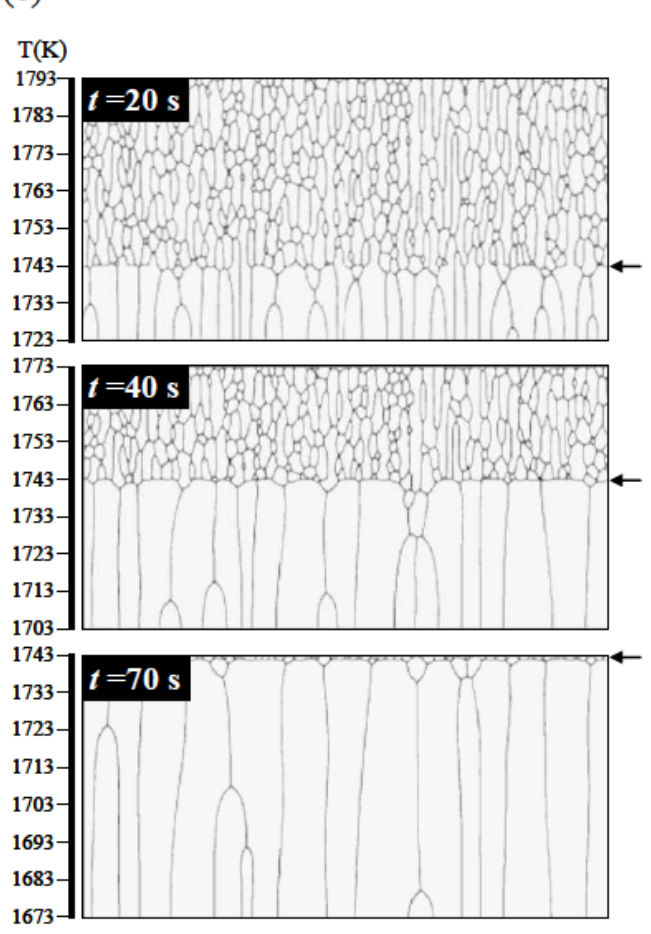

Fig. 2. (a) Example of initial grain structure $\left(d_{\text {initial }}=150 \mu \mathrm{m}\right.$ and $\left.a_{\text {initial }}=3\right)$ used in the present study. Temporal evolution processes of grain structures (from upper to lower snapshots) calculated for $\dot{T}=1.0 \mathrm{~K} / \mathrm{s}, T_{\gamma}=1743 \mathrm{~K}, d_{\text {initial }}=150 \mu \mathrm{m}, a_{\text {initial }}=3$, (b) $G=6.0 \times 10^{3} \mathrm{~K} / \mathrm{m}$ and (c) $G=1.4 \times 10^{4} \mathrm{~K} / \mathrm{m}$. The temperature is specified on the left-hand axis and the position of $T_{\gamma}$ is indicated by the arrow on the right-hand side in figures (b) and (c). 


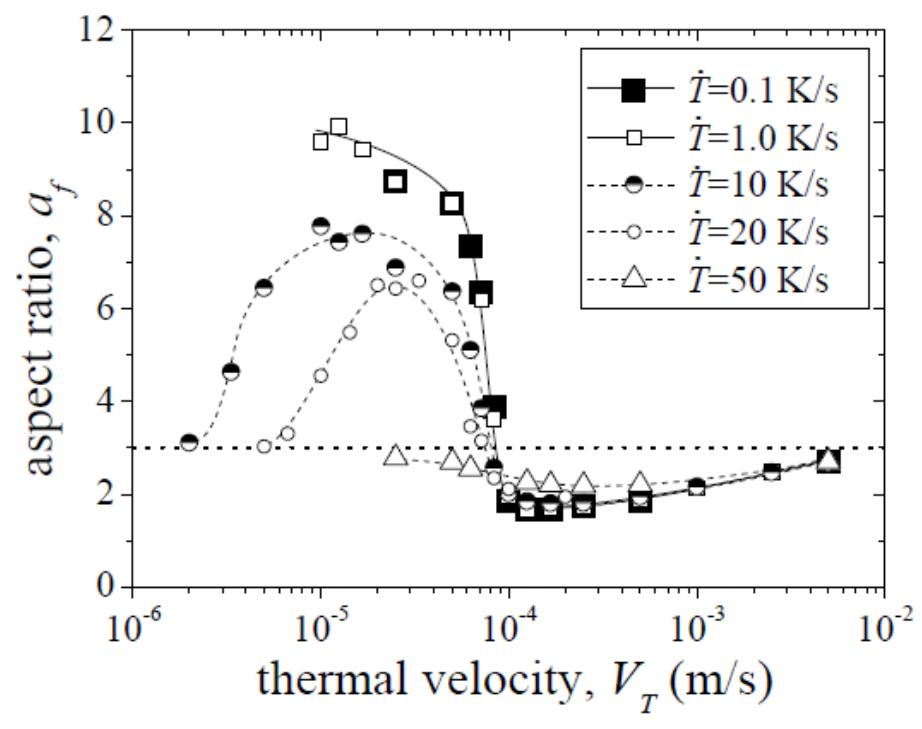

Fig. 3. Dependence of aspect ratio of the final grain structures on thermal velocity, $V_{T}=\dot{T} / G$, calculated for $T_{\gamma}=1743 \mathrm{~K}, d_{\text {initial }}=150 \mu \mathrm{m}$ and $a_{\text {initial }}=3$. The dotted horizontal line represents the initial aspect ratio. The solid lines for $\dot{T}=0.1$ and $1.0 \mathrm{~K} / \mathrm{s}$ are almost superimposed on each other. 


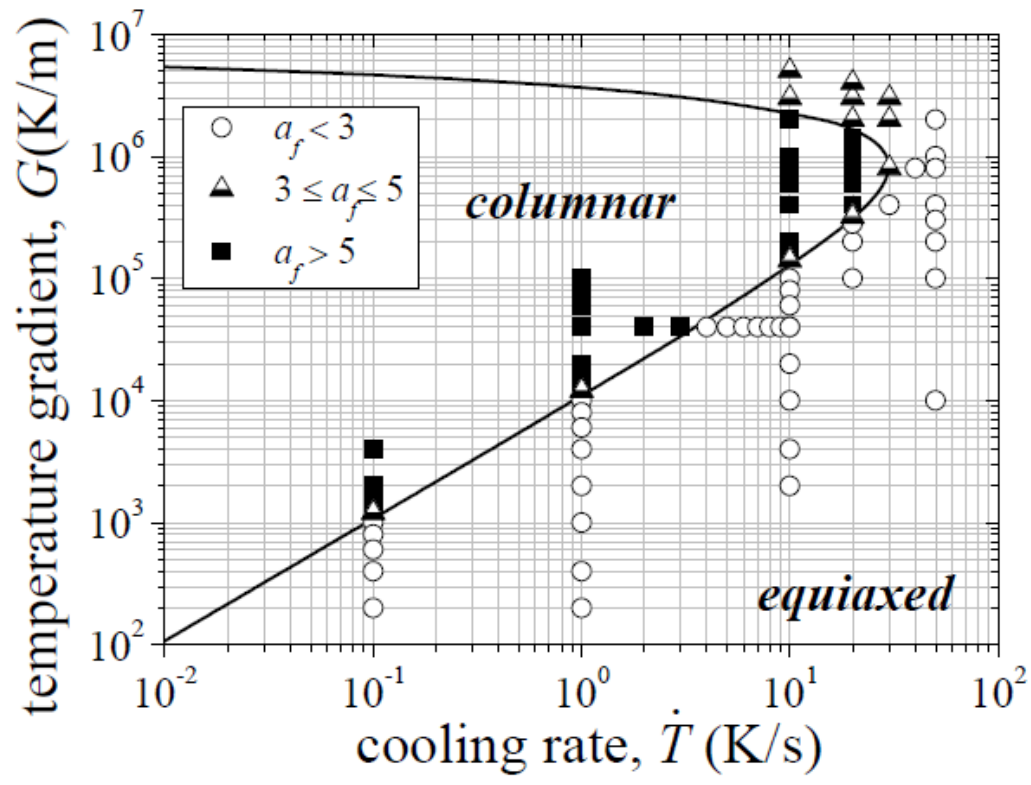

Fig. 4. Structure map for columnar and equiaxed grain structures calculated for $T_{\gamma}=1743$ $\mathrm{K}, d_{\text {initial }}=150 \mu \mathrm{m}, a_{\text {initial }}=3$. The plots indicate the results of phase-field simulations. The solid line is the calculated result of eq.(7). 


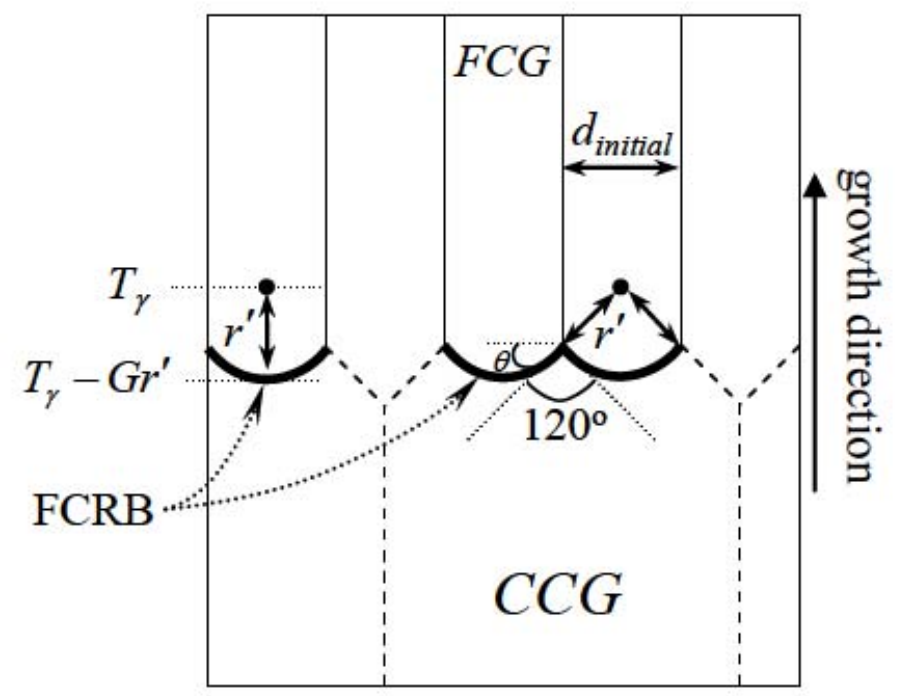

Fig. 5. Schematic illustration of FCG/CCG region boundary (FCRB). 
(a)

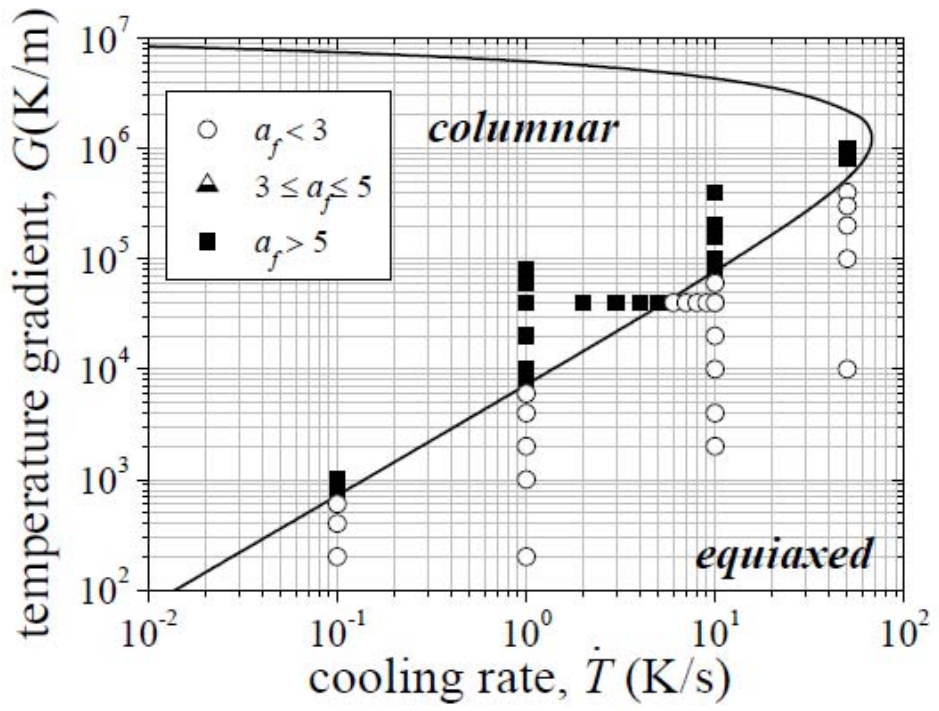

(b)

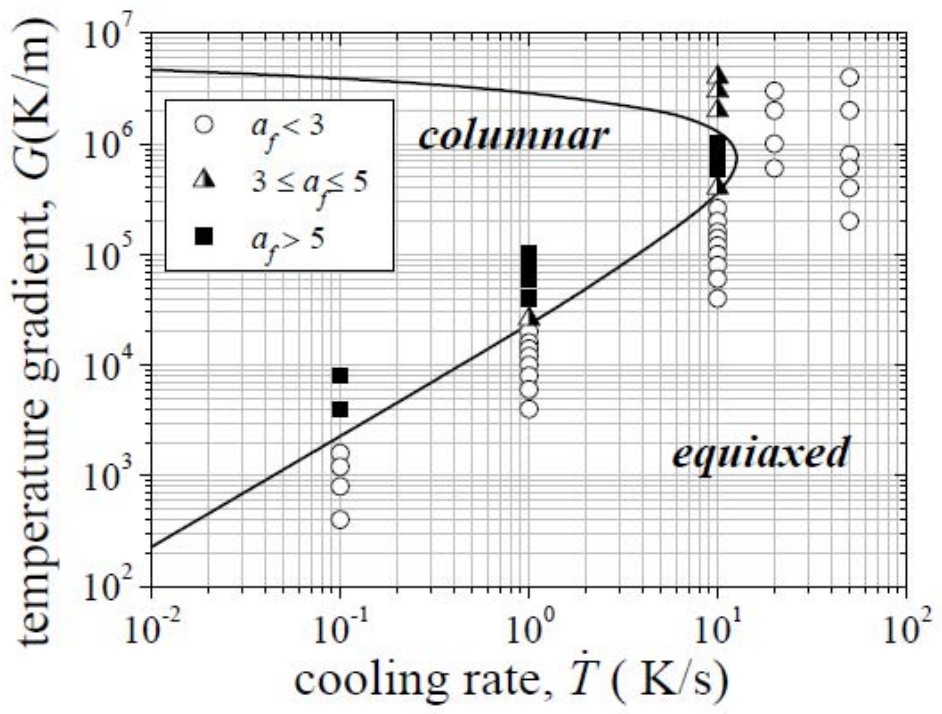

Fig. 6. Structure maps for (a) $T_{\gamma}=1743 \mathrm{~K}, d_{\text {initial }}=100 \mu \mathrm{m}, a_{\text {initial }}=3$, (b) $T_{\gamma}=1643 \mathrm{~K}$, $d_{\text {initial }}=150 \mu \mathrm{m}, a_{\text {initial }}=3$. The solid curve in each figure indicates the relation for $V_{T}=V_{\gamma}$. 

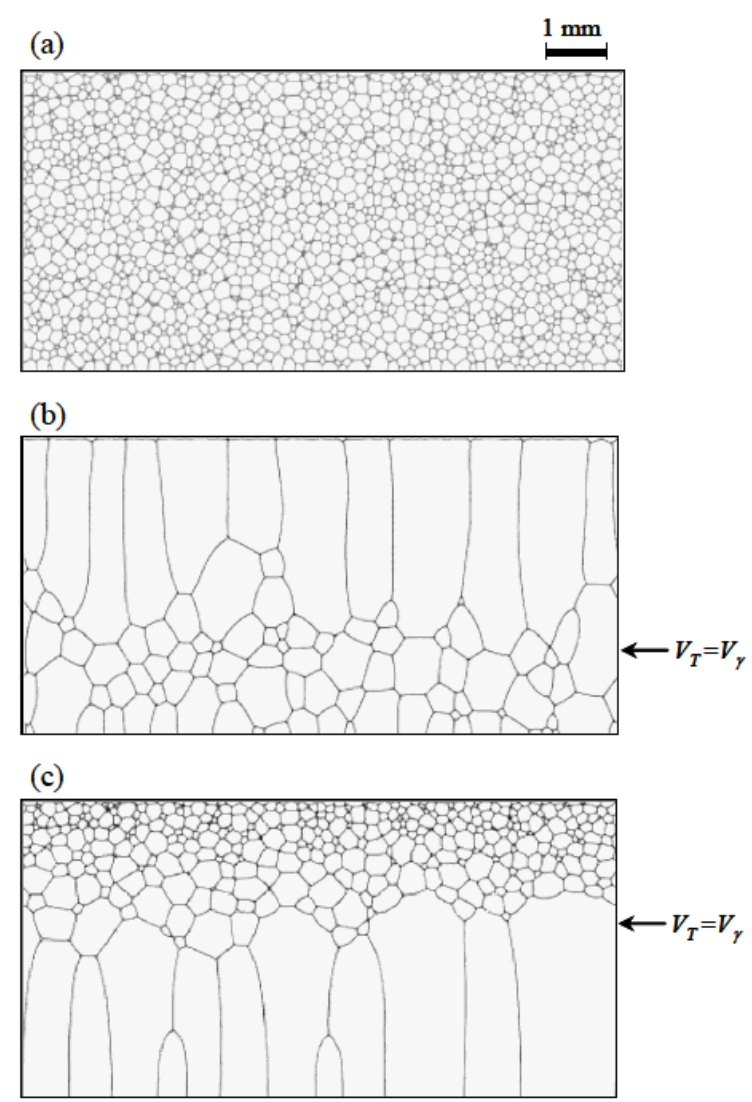

Fig. 7. (a) Initial grain structure $\left(d_{\text {initial }}=150 \mu \mathrm{m}\right.$ and $\left.a_{\text {initial }}=1\right)$. (b,c) Final grain structures calculated for $T_{\gamma}=1743 \mathrm{~K}$. (b) $G$ is kept to be $2.0 \times 10^{5} \mathrm{~K} / \mathrm{m}$ and $\dot{T}$ is varied with time from 20 to $5 \mathrm{~K} / \mathrm{s}$. (c) $\dot{T}$ is fixed to be $1.0 \mathrm{~K} / \mathrm{s}$ and $G$ is spatially varied from $1.6 \times 10^{4} \mathrm{~K} / \mathrm{m}$ at the bottom edge to $2.0 \times 10^{2} \mathrm{~K} / \mathrm{m}$ at the top edge. The position at which $V_{T}=V_{\gamma}$ is satisfied is indicated in each snapshot. 

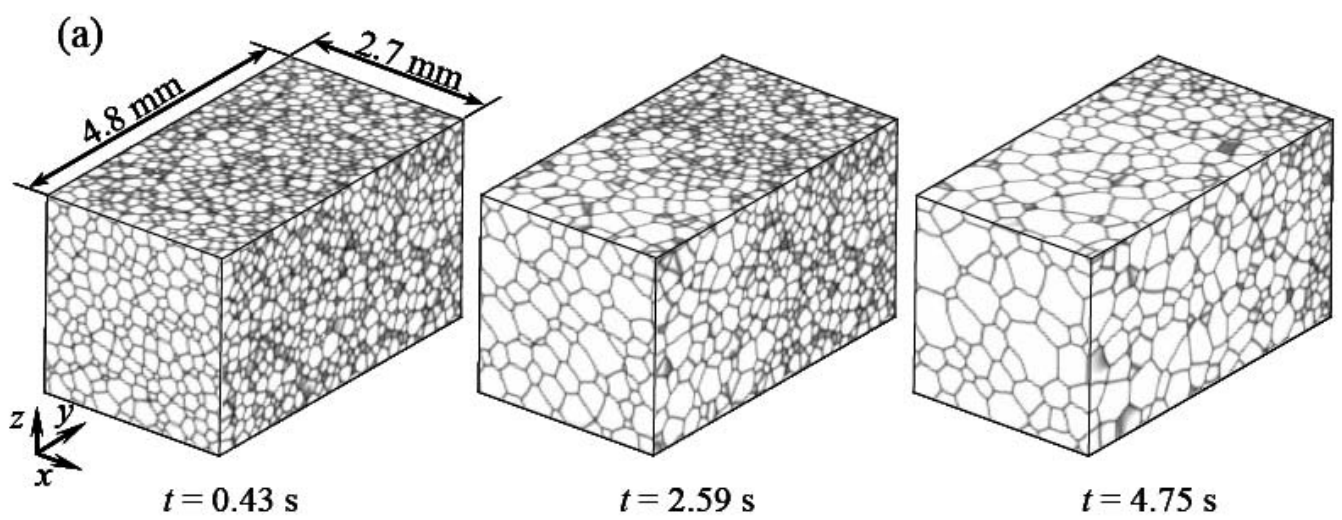

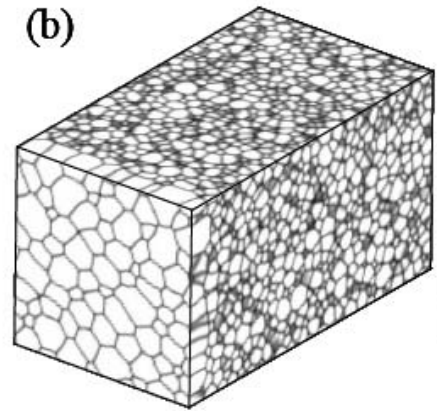

$t=4.3 \mathrm{~s}$

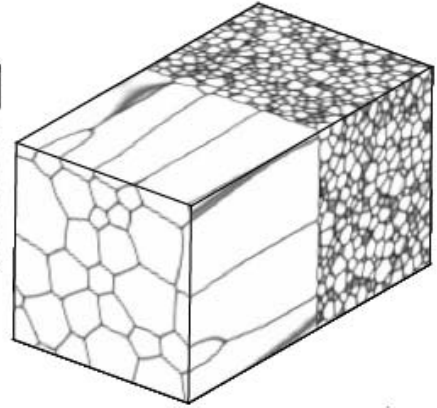

$t=25.9 \mathrm{~s}$

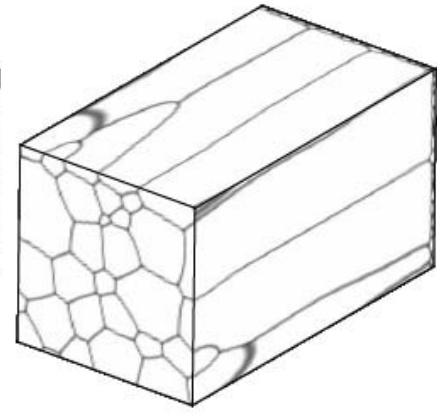

$t=47.5 \mathrm{~s}$

Fig. 8. Temporal evolutions of grain structures (from the left to right snapshots) calculated in $3 \mathrm{D}$ system for $T_{\gamma}=1743 \mathrm{~K}, d_{\text {initial }}=200 \mu \mathrm{m}, \dot{T}=10 \mathrm{~K} / \mathrm{s}$, (a) $G=1.0 \times 10^{4}$ $\mathrm{K} / \mathrm{m}$ and (b) $G=1.0 \times 10^{5} \mathrm{~K} / \mathrm{m}$. The temperature gradient is imposed along $y$-direction. 


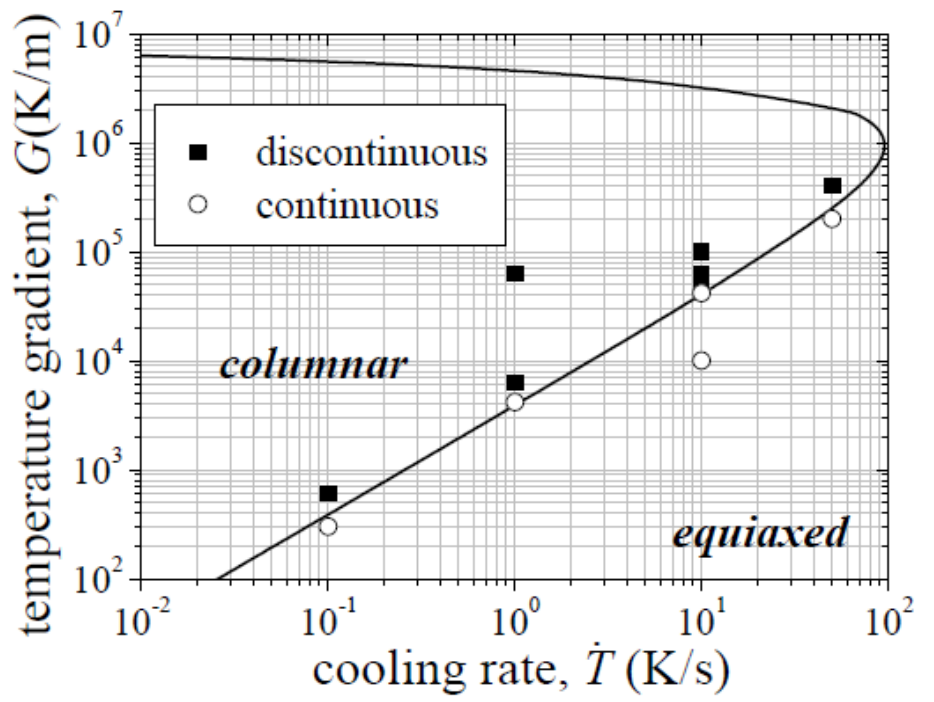

Fig. 9. Structure map for equiaxed and columnar grains calculated in 3D system for $T_{\gamma}=1743 \mathrm{~K}, d_{\text {initial }}=200 \mu \mathrm{m}$ and $a_{\text {initial }}=1$. The solid line indicates the relation for $V_{T}=V_{\gamma}$. 